\title{
Dynamical Systems Method for Solving Ill-conditioned Linear Algebraic Systems
}

\author{
Sapto W. Indratno \\ Department of Mathematics \\ Kansas State University, Manhattan, KS 66506-2602, USA \\ sapto@math.ksu.edu \\ A G Ramm \\ Department of Mathematics \\ Kansas State University, Manhattan, KS 66506-2602, USA \\ ramm@math.ksu. edu
}

\begin{abstract}
A new method, the Dynamical Systems Method (DSM), justified recently, is applied to solving ill-conditioned linear algebraic system (ICLAS). The DSM gives a new approach to solving a wide class of ill-posed problems. In this paper a new iterative scheme for solving ICLAS is proposed. This iterative scheme is based on the DSM solution. An a posteriori stopping rules for the proposed method is justified. This paper also gives an a posteriori stopping rule for a modified iterative scheme developed in A.G.Ramm, JMAA,330 (2007),1338-1346, and proves convergence of the solution obtained by the iterative scheme.
\end{abstract}

MSC: 15A12; 47A52; 65F05; 65F22

Keywords: Hilbert matrix, Fredholm integral equations of the first kind, iterative regularization, variational regularization, discrepancy principle, Dynamical Systems Method

Biographical notes: Professor Alexander G. Ramm is an author of more than 580 papers, 2 patents, 12 monographs, an editor of 3 books, and an associate editor of several mathematics and computational mathematics Journals. He gave more than 135 addresses at various Conferences, visited many Universities in Europe, Africa, America, Asia, and Australia. He won Khwarizmi Award in Mathematics, was Mercator Professor, Distinguished Visiting Professor supported by the Royal Academy of Engineering, invited plenary speaker at the Seventh PanAfrican Congress of Mathematicians, a London Mathematical Society speaker, distinguished HKSTAM speaker, CNRS research professor, Fulbright professor in Israel, distinguished Foreign professor in Mexico and 
Egypt. His research interests include inverse and ill-posed problems, scattering theory, wave propagation, mathematical physics, differential and integral equations, functional analysis, nonlinear analysis, theoretical numerical analysis, signal processing, applied mathematics and operator theory.

Sapto W. Indratno is currently a $\mathrm{PhD}$ student at Kansas State University under the supervision of Prof. Alexander G. Ramm. He is a coauthor of three accepted papers. His fields of interest are numerical analysis, optimization, stochastic processes, inverse and ill-posed problems, scattering theory, differential equations and applied mathematics.

\section{Introduction}

We consider a linear equation

$$
A u=f,
$$

where $A: \mathbb{R}^{m} \rightarrow \mathbb{R}^{m}$, and assume that equation (1) has a solution, possibly nonunique. According to Hadamard [9, p.9], problem (1) is called well-posed if the operator $A$ is injective, surjective, and $A^{-1}$ is continuous. Problem (1) is called ill-posed if it is not well-posed. Ill-conditioned linear algebraic systems arise as discretizations of ill-posed problems, such as Fredholm integral equations of the first kind,

$$
\int_{a}^{b} k(x, t) u(t) d t=f(x), c \leq x \leq d,
$$

where $k(x, t)$ is a smooth kernel. Therefore, it is of interest to develop a method for solving ill-conditioned linear algebraic systems stably. In this paper we give a method for solving linear algebraic systems (1) with an ill-conditioned-matrix $A$. The matrix $A$ is called ill-conditioned if $\kappa(A)>>1$, where $\kappa(A):=\|A\| \mid A^{-1} \|$ is the condition number of $A$. If the null-space of $A, \mathcal{N}(A):=\{u: A u=0\}$, is nontrivial, then $\kappa(A)=\infty$. Let $A=U \Sigma V^{*}$ be the singular value decomposition (SVD) of $A, U U^{*}=U^{*} U=I, V V^{*}=V^{*} V=I$, and $\Sigma=\operatorname{diag}\left(\sigma_{1}, \sigma_{2}, \ldots, \sigma_{m}\right)$, where $\sigma_{1} \geq \sigma_{2} \geq \cdots \geq \sigma_{m} \geq 0$ are the singular values of $A$. Applying this SVD to the matrix $A$ in (1), one gets

$$
f=\sum_{i} \beta_{i} u_{i} \text { and } y=\sum_{i, \sigma_{i}>0} \frac{\beta_{i}}{\sigma_{i}} v_{i},
$$

where $\beta_{i}=\left\langle u_{i}, f\right\rangle$. Here $\langle\cdot, \cdot\rangle$ denotes the inner product of two vectors. The terms with small singular values $\sigma_{i}$ in (3) cause instability of the solution, because the coefficients $\beta_{i}$ are known with errors. This difficulty is essential when one deals with an ill-conditioned matrix $A$. Therefore a regularization is needed for solving ill-conditioned linear algebraic system (1). There are many methods to solve (1) stably: variational regularization, quasisolutions, iterative regularization (see e.g, [2], [4], [6], [9]). The method proposed in this paper is based on the Dynamical Systems Method (DSM) developed in [9, p.76]. The DSM for 
solving equation (1) with, possibly, nonlinear operator $A$ consists of solving the Cauchy problem

$$
\dot{u}(t)=\Phi(t, u(t)), \quad u(0)=u_{0} ; \dot{u}(t):=\frac{d u}{d t},
$$

where $u_{0} \in H$ is an arbitrary element of a Hilbert space $H$, and $\Phi$ is some nonlinearity, chosen so that the following three conditions hold: a) there exists a unique solution $u(t) \quad \forall t \geq 0$, b) there exists $u(\infty)$, and c) $A u(\infty)=f$.

In this paper we choose $\Phi(t, u(t))=\left(A^{*} A+a(t) I\right)^{-1} f-u(t)$ and consider the following Cauchy problem:

$$
\dot{u}_{a}(t)=-u_{a}(t)+\left[A^{*} A+a(t) I_{m}\right]^{-1} A^{*} f, \quad u_{a}(0)=u_{0},
$$

where

$$
a(t)>0 \text {, and } a(t) \searrow 0 \text { as } t \rightarrow \infty,
$$

$A^{*}$ is the adjoint matrix and $I_{n}$ is an $m \times m$ identity matrix. The initial element $u_{0}$ in (5) can be chosen arbitrarily in $N(A)^{\perp}$, where

$$
\mathcal{N}(A):=\{u \mid A u=0\} .
$$

For example, one may take $u_{0}=0$ in (5) and then the unique solution to (5) with $u(0)=0$ has the form

$$
u(t)=\int_{0}^{t} e^{-(t-s)} T_{a(s)}^{-1} A^{*} f d s,
$$

where $T:=A^{*} A, T_{a}:=T+a I, I$ is the identity operator. In the case of noisy data we replace the exact data $f$ with the noisy data $f_{\delta}$ in (8), i.e.,

$$
u^{\delta}(t)=\int_{0}^{t_{\delta}} e^{-\left(t_{\delta}-s\right)} T_{a(s)}^{-1} A^{*} f_{\delta} d s,
$$

where $t_{\delta}$ is the stopping time which will be discussed later. There are many ways to solve the Cauchy problem (5). For example, one may apply a family of Runge-Kutta methods for solving (5). Numerically, the Runge-Kutta methods require an appropriate stepsize to get an accurate and stable solution. Usually the stepsizes have to be chosen sufficiently small to get such a solution. The number of steps will increase when $t_{\delta}$, the stopping time, increases, see [2]. Therefore the computation time will increase significantly. Since $\lim _{\delta \rightarrow 0} t_{\delta}=\infty$, as was proved in [9], the family of the Runge-Kutta method may be less efficient for solving the Cauchy problem (5) than the method, proposed in this paper. We give a simple iterative scheme, based on the DSM, which produces stable solution to equation (1). The novel points in our paper are iterative schemes (12) and (13) (see below), which are constructed on the basis of formulas (8) and (9), and a modification of the iterative scheme given in [8]. Our stopping rule for the iterative scheme (13) is given in (85) (see below). In [9, p.76] the function $a(t)$ is assumed to be a slowly decaying monotone function. In this 
paper instead of using the slowly decaying continuous function $a(t)$ we use the following piecewise-constant function:

$$
a^{(n)}(t)=\sum_{j=0}^{n-1} \alpha_{0} q^{j+1} \chi_{\left(t_{j}, t_{j+1}\right]}(t), \quad q \in(0,1), \quad t_{j}=-j \ln (q), n \in \mathbb{N},
$$

where $\mathbb{N}$ is the set of positive integer, $t_{0}=0, \alpha_{0}>0$, and

$$
\chi_{\left(t_{j}, t_{j+1}\right]}(t)= \begin{cases}1, & t \in\left(t_{j}, t_{j+1}\right] \\ 0, & \text { otherwise }\end{cases}
$$

The parameter $\alpha_{0}$ in (10) is chosen so that assumption (17) (see below) holds. This assumption plays an important role in the proposed iterative scheme. Definition (10) allows one to write (8) in the form

$$
u_{n+1}=q u_{n}+(1-q) T_{\alpha_{0} q^{n+1}}^{-1} A^{*} f, \quad u_{0}=0 .
$$

A detailed derivation of the iterative scheme (12) is given in Section 2. When the data $f$ are contaminated by some noise, we use $f_{\delta}$ in place of $f$ in (8), and get the iterative scheme

$$
u_{n+1}^{\delta}=q u_{n}^{\delta}+(1-q) T_{\alpha_{0} q^{n+1}}^{-1} A^{*} f_{\delta}, \quad u_{0}^{\delta}=0 .
$$

We always assume that

$$
\left\|f_{\delta}-f\right\| \leq \delta
$$

where $f_{\delta}$ are the noisy data, which are known, while $f$ is unknown, and $\delta$ is the level of noise. Here and throughout this paper the notation $\|z\|$ denotes the $l^{2}$-norm of the vector $z \in \mathbb{R}^{m}$. In this paper a discrepancy type principle (DP) is proposed to choose the stopping index of iteration (13). This DP is based on discrepancy principle for the DSM developed in [11], where the stopping time $t_{\delta}$ is obtained by solving the following nonlinear equation

$$
\int_{0}^{t_{\delta}} e^{-\left(t_{\delta}-s\right)} a(s)\left\|Q_{a(s)}^{-1} f_{\delta}\right\| d s=C \delta, \quad C \in(1,2] .
$$

It is a non-trivial task to obtain the stopping time $t_{\delta}$ satisfying (15). In this paper we propose a discrepancy type principle based on (15) which can be easily implemented numerically: iterative scheme (13) is stopped at the first integer $n_{\delta}$ satisfying the inequalities:

$$
\begin{aligned}
& \sum_{j=0}^{n_{\delta}-1}\left(q^{n_{\delta}-j-1}-q^{n-j}\right) \alpha_{0} q^{j+1}\left\|Q_{\alpha_{0} q^{j+1}}^{-1} f_{\delta}\right\| d s \leq C \delta^{\varepsilon} \\
& <\sum_{j=0}^{n-1}\left(q^{n-j-1}-q^{n-j}\right) \alpha_{0} q^{j+1}\left\|Q_{\alpha_{0} q^{j+1}}^{-1} f_{\delta}\right\|, 1 \leq n<n_{\delta},
\end{aligned}
$$


and it is assumed that

$$
(1-q) \alpha_{0} q\left\|Q_{\alpha_{0} q}^{-1} f_{\delta}\right\| \geq C \delta^{\varepsilon}, \quad C>1, \quad \varepsilon \in(0,1), \quad \alpha_{0}>0 .
$$

We prove in Section 2 that using discrepancy-type principle (16), one gets the convergence:

$$
\lim _{\delta \rightarrow 0}\left\|u_{n_{\delta}}^{\delta}-y\right\|=0
$$

where $u_{n}^{\delta}$ is defined in (13). About other versions of discrepancy principles for DSM we refer the reader to [6],[12]. In this paper we assume that $A$ is bounded. If the operator $A$ is unbounded then $f_{\delta}$ may not belong to the domain of $A^{*}$. In this case the expression $A^{*} f_{\delta}$ is not defined. In [7], [8] and [10] solving (1) with unbounded operators is discussed. In these papers the unbounded operator $A$ is assumed to be linear, closed, densely defined operator in a Hilbert space. Under these assumptions one may use the operator $A^{*}\left(A A^{*}+a I\right)^{-1}$ in place of $T_{a}^{-1} A^{*}$. This operator is defined for any $f$ in the Hilbert space.

In [8] an iterative scheme with a constant regularization parameter is given:

$$
u_{n+1}^{\delta}=a T_{a}^{-1} u_{n}^{\delta}+T_{a}^{-1} A^{*} f_{\delta},
$$

but the stopping rule, which produces a stable solution of equation (1) by this iterative scheme, has not been discussed in [8]. In this paper the constant regularization parameter $a$ in iterative scheme (19) is replaced with the geometric series $\left\{\alpha_{0} q^{n}\right\}_{n=1}^{\infty}, \alpha_{0}>0, q \in(0,1)$, i.e.

$$
u_{n+1}^{\delta}=\alpha_{0} q^{n} T_{\alpha_{0} q^{n}}^{-1} u_{n}^{\delta}+T_{\alpha_{0} q^{n}}^{-1} A^{*} f_{\delta} .
$$

Stopping rule (85) (see below) is used for this iterative scheme. Without loss of generality we use $\alpha_{0}=1$ in (20). The convergence analysis of this iterative scheme is presented in Section 3. In Section 4 some numerical experiments are given to illustrate the efficiency of the proposed methods.

\section{Derivation of the proposed method}

In this section we give a detailed derivation of iterative schemes (12) and (13). Let us denote by $y \in \mathbb{R}^{m}$ the unique minimal-norm solution of equation (1). Throughout this paper we denote $T_{a(t)}:=A^{*} A+a(t) I_{m}$, where $I_{m}$ is the identity operator in $\mathbb{R}^{m}$, and $a(t)$ is given in (10).

Lemma 2.1. Let $g(x)$ be a continuous function on $(0, \infty), c>0$ and $q \in(0,1)$ be constants. If

$$
\lim _{x \rightarrow 0^{+}} g(x)=g(0):=g_{0},
$$

then

$$
\lim _{n \rightarrow \infty} \sum_{j=1}^{n-1}\left(q^{n-j-1}-q^{n-j}\right) g\left(c q^{j+1}\right)=g_{0} .
$$


Proof. Let

$$
\omega_{j}(n):=q^{n-j}-q^{n+1-j}, \quad \omega_{j}(n)>0,
$$

and

$$
F_{l}(n):=\sum_{j=1}^{l-1} \omega_{j}(n) g\left(c q^{j}\right)
$$

Then

$$
\left|F_{n+1}(n)-g_{0}\right| \leq\left|F_{l}(n)\right|+\left|\sum_{j=l}^{n} \omega_{j}(n) g\left(c q^{j}\right)-g_{0}\right| .
$$

Take $\epsilon>0$ arbitrary small. For sufficiently large $l(\epsilon)$ one can choose $n(\epsilon)$, such that

$$
\left|F_{l(\epsilon)}(n)\right| \leq \frac{\epsilon}{2}, \forall n>n(\epsilon),
$$

because $\lim _{n \rightarrow \infty} q^{n}=0$. Fix $l=l(\epsilon)$ such that $\left|g\left(c q^{j}\right)-g_{0}\right| \leq \frac{\epsilon}{2}$ for $j>l(\epsilon)$. This is possible because of (21). One has

$$
\left|F_{l(\epsilon)}(n)\right| \leq \frac{\epsilon}{2}, n>n(\epsilon)
$$

and

$$
\begin{aligned}
\left|\sum_{j=l(\epsilon)}^{n} \omega_{j}(n) g\left(c q^{j}\right)-g_{0}\right| & \leq \sum_{j=l(\epsilon)}^{n} \omega_{j}(n)\left|g\left(c q^{j}\right)-g_{0}\right|+\left|\sum_{j=l(\epsilon)}^{n} \omega_{j}(n)-1\right|\left|g_{0}\right| \\
& \leq \frac{\epsilon}{2} \sum_{j=l(\epsilon)}^{n} \omega_{j}(n)+q^{n-l(\epsilon)}\left|g_{0}\right| \\
& \leq \frac{\epsilon}{2}+\left|g_{0}\right| q^{n-l(\epsilon)} \leq \epsilon,
\end{aligned}
$$

if $n$ is sufficiently large. Here we have used the relation

$$
\sum_{j=l}^{n} \omega_{j}(n)=1-q^{n+1-l}
$$

Since $\epsilon>0$ is arbitrarily small, Lemma 2.1 is proved.

Let us define

$$
u_{n}:=\int_{0}^{t_{n}} e^{-\left(t_{n}-s\right)} T_{a^{(n)}(s)}^{-1} A^{*} f d s, \quad t_{n}=-n \ln (q), \quad q \in(0,1) .
$$


Note that

$$
\begin{aligned}
u_{n} & =\int_{0}^{t_{n-1}} e^{-\left(t_{n}-s\right)} T_{a^{(n)}(s)}^{-1} A^{*} f d s+\int_{t_{n-1}}^{t_{n}} e^{-\left(t_{n}-s\right)} T_{a^{(n)}(s)}^{-1} A^{*} f d s \\
& =e^{-\left(t_{n}-t_{n-1}\right)} \int_{0}^{t_{n-1}} e^{-\left(t_{n-1}-s\right)} T_{a(s)}^{-1} A^{*} f d s+\int_{t_{n-1}}^{t_{n}} e^{-\left(t_{n}-s\right)} T_{a^{(n)}(s)}^{-1} A^{*} f d s \\
& =e^{-\left(t_{n}-t_{n-1}\right)} u_{n-1}+\int_{t_{n-1}}^{t_{n}} e^{-\left(t_{n}-s\right)} T_{a^{(n)}(s)}^{-1} A^{*} f d s .
\end{aligned}
$$

Using definition (10), one gets

$$
\begin{aligned}
u_{n} & =e^{-\left(t_{n}-t_{n-1}\right)} u_{n-1}+\left[1-e^{-\left(t_{n}-t_{n-1}\right)}\right] T_{\alpha_{0} q^{n}}^{-1} A^{*} f \\
& =\frac{q^{n}}{q^{n-1}} u_{n-1}+\left(1-\frac{q^{n}}{q^{n-1}}\right) T_{\alpha_{0} q^{n}}^{-1} A^{*} f .
\end{aligned}
$$

Therefore, (25) can be rewritten as iterative scheme (12).

Lemma 2.2. Let $u_{n}$ be defined in (12) and $A y=f$. Then

$$
\left\|u_{n}-y\right\| \leq q^{n}\|y\|+\sum_{j=0}^{n-1}\left(q^{n-j-1}-q^{n-j}\right) \alpha_{0} q^{j+1}\left\|T_{\alpha_{0} q^{j+1}}^{-1} y\right\|, \quad \forall n \geq 1,
$$

and

$$
\left\|u_{n}-y\right\| \rightarrow 0 \text { as } n \rightarrow \infty .
$$

Proof. By definitions (25) and (10) we obtain

$$
u_{n}=\int_{0}^{t_{n}} e^{-\left(t_{n}-s\right)} T_{a(s)}^{-1} A^{*} f d s=\sum_{j=0}^{n-1}\left(\frac{q^{n}}{q^{j+1}}-\frac{q^{n}}{q^{j}}\right) T_{\alpha_{0} q^{j+1}}^{-1} A^{*} f .
$$

From (28) and the equation $A y=f$, one gets:

$$
\begin{aligned}
u_{n} & =\sum_{j=0}^{n-1}\left(\frac{q^{n}}{q^{j+1}}-\frac{q^{n}}{q^{j}}\right) T_{\alpha_{0} q^{j+1}}^{-1} A^{*} f \\
& =\sum_{j=0}^{n-1}\left(\frac{q^{n}}{q^{j+1}}-\frac{q^{n}}{q^{j}}\right) T_{\alpha_{0} q^{j+1}}^{-1}\left(T_{\alpha_{0} q^{j+1}}-\alpha_{0} q^{j+1} I_{m}\right) y \\
& =\sum_{j=0}^{n-1}\left(q^{n-j-1}-q^{n-j}\right) y-\sum_{j=0}^{n-1}\left(q^{n-j-1}-q^{n-j}\right) \alpha_{0} q^{j+1} T_{\alpha_{0} q^{j+1}}^{-1} y \\
& =y-q^{n} y-\sum_{j=0}^{n-1}\left(q^{n-j-1}-q^{n-j}\right) \alpha_{0} q^{j+1} T_{\alpha_{0} q^{j+1}}^{-1} y .
\end{aligned}
$$

Thus, estimate (26) follows. To prove (27), we apply Lemma 2.1 with $g(a):=$ $a\left\|T_{a}^{-1} y\right\|$. Since $y \perp \mathcal{N}(A)$, it follows from the spectral theorem that

$$
\lim _{a \rightarrow 0} g^{2}(a)=\lim _{a \rightarrow 0} \int_{0}^{\infty} \frac{a^{2}}{(a+s)^{2}} d\left\langle E_{s} y, y\right\rangle=\left\|P_{\mathcal{N}(A)} y\right\|^{2}=0,
$$


where $E_{s}$ is the resolution of the identity corresponding to $A^{*} A$, and $P$ is the orthogonal projector onto $\mathcal{N}(A)$. Thus, by Lemma $2.1,(27)$ follows.

Let us discuss iterative scheme (13). The following lemma gives the estimate of the difference of the solutions $u_{n}^{\delta}$ and $u_{n}$.

Lemma 2.3. Let $u_{n}$ and $u_{n}^{\delta}$ be defined in (12) and (13), respectively. Then

$$
\left\|u_{n}^{\delta}-u_{n}\right\| \leq \frac{\sqrt{q}}{1-q^{3 / 2}} w_{n}, \quad n \geq 0
$$

where $w_{n}:=(1-q) \frac{\delta}{2 \sqrt{q} \sqrt{\alpha_{0} q^{n}}}$.

Proof. Let $H_{n}:=\left\|u_{n}^{\delta}-u_{n}\right\|$. Then from the definitions of $u_{n}^{\delta}$ and $u_{n}$ we get the estimate

$$
H_{n+1} \leq q\left\|u_{n}^{\delta}-u_{n}\right\|+(1-q)\left\|T_{\alpha_{0} q^{n+1}}^{-1} A^{*}\left(f_{\delta}-f\right)\right\| \leq q H_{n}+w_{n}
$$

Let us prove inequality (29) by induction. For $n=0$ one has $u_{0}=u_{0}^{\delta}=0$, so (29) holds. For $n=1$ one has $\left\|u_{1}^{\delta}-u_{1}\right\| \leq(1-q) \frac{\delta}{2 \sqrt{\alpha_{0} q^{2}}}$, so (29) holds. If (29) holds for $n \leq k$, then for $n=k+1$ one has

$$
\begin{aligned}
H_{k+1} & \leq q H_{k}+w_{k} \leq\left(\frac{q^{3 / 2}}{1-q^{3 / 2}}+1\right) w_{k}=\frac{1}{1-q^{3 / 2}} w_{k} \\
& =\frac{1}{1-q^{3 / 2}} \frac{w_{k}}{w_{k+1}} w_{k+1} \leq \frac{1}{1-q^{3 / 2}} \sqrt{q} w_{k+1} .
\end{aligned}
$$

Hence (29) is proved for $n \geq 0$.

\subsection{Stopping criterion}

In this section we give a stopping rule for iterative scheme given in (13). Let $Q:=A A^{*}, Q_{a}:=Q+a I_{m}$, and

$$
\begin{aligned}
G_{n} & :=\int_{0}^{t_{n}} e^{-\left(t_{n}-s\right)} a(s)\left\|Q_{a(s)}^{-1} f_{\delta}\right\| d s \\
& =\sum_{j=0}^{n-1}\left(q^{n-j-1}-q^{n-j}\right) \alpha_{0} q^{j+1}\left\|Q_{\alpha_{0} q^{j+1}}^{-1} f_{\delta}\right\|, \quad n \geq 1,
\end{aligned}
$$

where $t_{n}=-n \ln q, q \in(0,1)$ and $\alpha_{0}>0$. Then stopping rule (16) can be rewritten as

$$
G_{n_{\delta}} \leq C \delta^{\varepsilon}<G_{n}, \quad 1 \leq n<n_{\delta}, \quad \varepsilon \in(0,1), \quad C>1, \quad G_{1}>C \delta^{\varepsilon} .
$$


Note that

$$
\begin{aligned}
G_{n+1} & =\sum_{j=0}^{n}\left(q^{n-j}-q^{n+1-j}\right) \alpha_{0} q^{j+1}\left\|Q_{\alpha_{0} q^{j+1}}^{-1} f_{\delta}\right\| \\
& =\sum_{j=0}^{n-1}\left(q^{n-j}-q^{n+1-j}\right) \alpha_{0} q^{j+1}\left\|Q_{\alpha_{0} q^{j+1}}^{-1} f_{\delta}\right\|+(1-q) \alpha_{0} q^{n+1}\left\|Q_{\alpha_{0} q^{n+1}}^{-1} f_{\delta}\right\| \\
& =q G_{n}+(1-q) \alpha_{0} q^{n+1}\left\|Q_{\alpha_{0} q^{n+1}}^{-1} f_{\delta}\right\|
\end{aligned}
$$

so

$$
G_{n}=q G_{n-1}+(1-q) \alpha_{0} q^{n}\left\|Q_{\alpha_{0} q^{n}}^{-1} f_{\delta}\right\|, \quad n \geq 1, \quad G_{0}=0 .
$$

Lemma 2.4. Let $G_{n}$ be defined in (34). Then

$$
G_{n} \leq \frac{1}{1-\sqrt{q}} \sqrt{\alpha_{0} q^{n}} \frac{\|y\|}{2}+\delta, \quad n \geq 1, \quad q \in(0,1) .
$$

Proof. Using the identity

$$
-a Q_{a}^{-1}=A T_{a}^{-1} A^{*}-I_{m}, a>0, T:=A^{*} A, T_{a}:=T+a I_{m},
$$

the estimates

$$
a\left\|Q_{a}^{-1}\right\| \leq 1,\left\|f_{\delta}-f\right\| \leq \delta
$$

and

$$
a\left\|A T_{a}^{-1}\right\| \leq \frac{\sqrt{a}}{2},
$$

where $Q:=A A^{*}, Q_{a}:=Q+a I_{m}$, we get

$$
\begin{aligned}
G_{n} & =q G_{n-1}+(1-q)\left\|A T_{\alpha_{0} q^{n}}^{-1} A^{*} f_{\delta}-f_{\delta}\right\| \\
& =q G_{n-1}+(1-q)\left\|A A^{*} Q_{\alpha_{0} q^{n}}^{-1} f_{\delta}-f_{\delta}\right\| \\
& =q G_{n-1}+(1-q)\left\|\left(A A^{*}+\alpha_{0} q^{n} I-\alpha_{0} q^{n} I\right) Q_{q^{n}}^{-1} f_{\delta}-f_{\delta}\right\| \\
& =q G_{n-1}+(1-q) \alpha_{0} q^{n}\left\|Q_{\alpha_{0} q^{n}}^{-1} f_{\delta}\right\| \\
& =q G_{n-1}+(1-q) \alpha_{0} q^{n}\left\|Q_{\alpha_{0} q^{n}}^{-1}\left(f_{\delta}-f+f\right)\right\| \\
& \leq q G_{n-1}+(1-q) \alpha_{0} q^{n}\left\|Q_{\alpha_{0} q^{n}}^{-1}\left(f_{\delta}-f\right)\right\|+(1-q) \alpha_{0} q^{n}\left\|Q_{\alpha_{0} q^{n}}^{-1} f\right\| \\
& \leq q G_{n-1}+(1-q) \delta+(1-q)\left\|A T_{\alpha_{0} q^{n}}^{-1} A^{*} f-f\right\| \\
& =q G_{n-1}+(1-q) \delta+(1-q)\left\|A\left(T_{\alpha_{0} q^{n}}^{-1} A^{*} A y-y\right)\right\| \\
& =q G_{n-1}+(1-q) \delta+(1-q)\left\|A\left(-\alpha_{0} q^{n} T_{\alpha_{0} q^{n}}^{-1} y\right)\right\| \\
& =q G_{n-1}+(1-q) \delta+(1-q) \alpha_{0} q^{n}\left\|A T_{\alpha_{0} q^{n}}^{-y}\right\| \\
& \leq q G_{n-1}+(1-q) \delta+(1-q) \alpha_{0} q^{n} \frac{\|y\|}{2 \sqrt{\alpha_{0} q^{n}}} \\
& =q G_{n-1}+(1-q) \delta+(1-q) \sqrt{\alpha_{0} q^{n}} \frac{\|y\|}{2} \\
& =q G_{n-1}+(1-q) \delta+\sqrt{q} \frac{\sqrt{\alpha_{0} q^{n-1}}}{2}\|y\| .
\end{aligned}
$$


Therefore,

$$
G_{n}-\delta \leq q\left(G_{n-1}-\delta\right)+\sqrt{q} \frac{\sqrt{\alpha_{0} q^{n-1}}}{2}\|y\|, \quad n \geq 1, G_{0}=0 .
$$

Let us prove relation (35) by induction. From relation (37) we get

$$
G_{1}-\delta \leq-q \delta+\frac{\sqrt{\alpha_{0} q}}{2}\|y\| \leq-q \delta+\frac{1}{1-\sqrt{q}} \frac{\sqrt{\alpha_{0} q}}{2}\|y\| \leq \frac{1}{1-\sqrt{q}} \frac{\sqrt{\alpha_{0} q}}{2}\|y\| .
$$

Thus, for $n=1$ relation (35) holds. Suppose that

$$
G_{n}-\delta \leq \frac{1}{1-\sqrt{q}} \frac{\sqrt{\alpha_{0} q^{n}}}{2}\|y\|, \quad 1 \leq n \leq k .
$$

Then by inequalities (37) and (39) we obtain

$$
\begin{aligned}
G_{k+1}-\delta & \leq q\left(G_{k}-\delta\right)+\sqrt{q} \frac{\sqrt{\alpha_{0} q^{k}}}{2}\|y\| \\
& \leq q \frac{1}{1-\sqrt{q}} \frac{\sqrt{\alpha_{0} q^{k}}}{2}\|y\|+\sqrt{q} \frac{\sqrt{\alpha_{0} q^{k}}}{2}\|y\| \\
& =\frac{\sqrt{q}}{1-\sqrt{q}} \frac{\sqrt{\alpha_{0} q^{k}}}{2}\|y\|=\frac{\sqrt{q}}{1-\sqrt{q}} \frac{\sqrt{\alpha_{0} q^{k}}}{2 \sqrt{\alpha_{0} q^{k+1}}} \sqrt{\alpha_{0} q^{k+1}}\|y\| \\
& \leq \frac{1}{1-\sqrt{q}} \frac{\sqrt{\alpha_{0} q^{k+1}}}{2}\|y\| .
\end{aligned}
$$

Thus, relation (35) is proved.

Lemma 2.5. Let $G_{n}$ be defined in (34), $q \in(0,1)$, and $\alpha_{0}>0$ be chosen such that $G_{1}>C \delta^{\varepsilon}, \varepsilon \in(0,1), C>1$. Then there exists a unique integer $n_{c}$ such that

$$
G_{n_{c}-1}<G_{n_{c}} \text { and } G_{n_{c}}>G_{n_{c}+1}, \quad n_{c} \geq 1
$$

Moreover,

$$
G_{n+1}<G_{n}, \quad \forall n \geq n_{c} .
$$

Proof. From Lemma 2.4 we have

$$
G_{n} \leq \frac{1}{1-\sqrt{q}} \sqrt{\alpha_{0} q^{n}} \frac{\|y\|}{2}+\delta, \quad n \geq 1, \quad q \in(0,1) .
$$

Since $G_{1}>C \delta^{\varepsilon}$ and $\lim \sup _{n \rightarrow \infty} G_{n} \leq \delta<C \delta^{\varepsilon}$, it follows that there exists an integer $n_{c} \geq 1$ such that $G_{n_{c}-1}<G_{n_{c}}$ and $G_{n_{c}}>G_{n_{c}+1}$. Let us prove the monotonicity of $G_{n}$, for $n \geq n_{c}$. We have $G_{n_{c}+1}-G_{n_{c}}<0$. Using definition (34), we get

$$
\begin{aligned}
G_{n_{c}+1}-G_{n_{c}} & =q G_{n_{c}}+(1-q) \alpha_{0} q^{n_{c}+1}\left\|Q_{\alpha_{0} q^{n_{c}+1}}^{-1} f_{\delta}\right\|-G_{n_{c}} \\
& =(1-q)\left(\alpha_{0} q^{n_{c}+1}\left\|Q_{\alpha_{0} q^{n_{c}+1}}^{-1} f_{\delta}\right\|-G_{n_{c}}\right)<0 .
\end{aligned}
$$


This implies

$$
\alpha_{0} q^{n_{c}+1}\left\|\alpha_{0} Q_{q^{n_{c}+1}}^{-1} f_{\delta}\right\|-G_{n_{c}}<0 .
$$

Note that

$$
G_{n+1}-G_{n}=(1-q)\left(\alpha_{0} q^{n+1}\left\|Q_{\alpha_{0} q^{n+1}}^{-1} f_{\delta}\right\|-G_{n}\right) .
$$

Therefore, to prove the monotonicity of $G_{n}$ for $n \geq n_{c}$, one needs to prove the inequality

$$
\alpha_{0} q^{n+1}\left\|Q_{\alpha_{0} q^{n+1}}^{-1} f_{\delta}\right\|-G_{n}<0, \quad \forall n \geq n_{c}
$$

This inequality is a consequence of the following lemma:

Lemma 2.6. Let $G_{n}$ be defined in (34), and (44) holds. Then

$$
\alpha_{0} q^{n+1}\left\|Q_{\alpha_{0} q^{n+1}}^{-1} f_{\delta}\right\|-G_{n}<0, \quad \forall n \geq n_{c} .
$$

Proof. Let us prove Lemma 2.6 by induction. Let

$$
D_{n}:=\alpha_{0} q^{n+1}\left\|Q_{\alpha_{0} q^{n+1}}^{-1} f_{\delta}\right\|-G_{n}
$$

and

$$
h(a):=a^{2}\left\|Q_{a}^{-1} f_{\delta}\right\|^{2} .
$$

The function $h(a)$ is a monotonically growing function of $a, a>0$. Indeed, by the spectral theorem, we get

$$
\begin{aligned}
h\left(a_{1}\right) & =a_{1}^{2}\left\|Q_{a_{1}}^{-1} f_{\delta}\right\|^{2}=\int_{0}^{\infty} \frac{a_{1}^{2}}{\left(a_{1}+s\right)^{2}} d\left\langle F_{s} f_{\delta}, f_{\delta}\right\rangle \\
& \leq \int_{0}^{\infty} \frac{a_{2}^{2}}{\left(a_{2}+s\right)^{2}} d\left\langle F_{s} f_{\delta}, f_{\delta}\right\rangle=a_{2}^{2}\left\|Q_{a_{2}}^{-1} f_{\delta}\right\|^{2}=h\left(a_{2}\right),
\end{aligned}
$$

where $F_{s}$ is the resolution of the identity corresponding to $Q:=A A^{*}$, because $\frac{a_{1}^{2}}{\left(a_{1}+s\right)^{2}} \leq \frac{a_{2}^{2}}{\left(a_{2}+s\right)^{2}}$ if $0<a_{1}<a_{2}$ and $s \geq 0$. By the assumption we have $D_{n_{c}}=\alpha_{0} q^{n_{c}+1}\left\|Q_{\alpha_{0} q^{n_{c}+1}}^{-1} f_{\delta}\right\|-G_{n_{c}}<0$. Thus, relation (45) holds for $n=n_{c}$. For $n=n_{c}+1$ we get

$$
\begin{aligned}
D_{n_{c}+1} & =\alpha_{0} q^{n_{c}+2}\left\|Q_{\alpha_{0} q^{n_{c}+2}}^{-1} f_{\delta}\right\|-(1-q) \alpha_{0} q^{n_{c}+1}\left\|Q_{\alpha_{0} q^{n_{c}+1}}^{-1} f_{\delta}\right\|-q G_{n_{c}} \\
& =\sqrt{h\left(\alpha_{0} q^{n_{c}+2}\right)}-\sqrt{h\left(\alpha_{0} q^{n_{c}+1}\right)}+q \sqrt{h\left(\alpha_{0} q^{n_{c}+1}\right)}-q G_{n_{c}} \\
& =\sqrt{h\left(\alpha_{0} q^{n_{c}+2}\right)}-\sqrt{h\left(\alpha_{0} q^{n_{c}+1}\right)}+q\left(\sqrt{h\left(\alpha_{0} q^{n_{c}+1}\right)}-G_{n_{c}}\right) \\
& =\sqrt{h\left(\alpha_{0} q^{n_{c}+2}\right)}-\sqrt{h\left(\alpha_{0} q^{n_{c}+1}\right)}+q D_{n_{c}} \\
& =\sqrt{h\left(\alpha_{0} q^{n_{c}+2}\right)}-\sqrt{h\left(\alpha_{0} q^{n_{c}+1}\right)}+q D_{n_{c}}<0 .
\end{aligned}
$$

Here we have used the monotonicity of the function $h(a)$. Thus, relation (45) holds for $n=n_{c}+1$. Suppose

$$
D_{n}<0, \quad n_{c} \leq n \leq n_{c}+k-1 .
$$


This, together with the monotonically growth of the function $h(a):=a^{2}\left\|Q_{q}^{-1} f_{\delta}\right\|^{2}$, yields

$$
\begin{aligned}
D_{n_{c}+k} & =\alpha_{0} q^{n_{c}+k+1}\left\|Q_{\alpha_{0} q^{n_{c}+k+1}}^{-1} f_{\delta}\right\|-G_{n_{c}+k} \\
& =\sqrt{h\left(\alpha_{0} q^{n_{c}+k+1}\right)}-(1-q) \sqrt{h\left(\alpha_{0} q^{n_{c}+k}\right)}-q G_{n_{c}+k-1} \\
& =\sqrt{h\left(\alpha_{0} q^{n_{c}+k+1}\right)}-\sqrt{h\left(\alpha_{0} q^{n_{c}+k}\right)}+q\left(\sqrt{h\left(\alpha_{0} q^{n_{c}+k}\right)}-G_{n_{c}+k-1}\right) \\
& =\sqrt{h\left(\alpha_{0} q^{n_{c}+k+1}\right)}-\sqrt{h\left(\alpha_{0} q^{n_{c}+k}\right)}+q D_{n_{c}+k-1} \\
& =\sqrt{h\left(\alpha_{0} q^{n_{c}+k+1}\right)}-\sqrt{h\left(\alpha_{0} q^{n_{c}+k}\right)}+q D_{n_{c}+k-1}<0 .
\end{aligned}
$$

Thus, $D_{n}<0, n \geq 1$. Lemma 2.6 is proved.

Let us continue with the proof of Lemma 2.5. From relation (34) we have

$$
\begin{aligned}
G_{n+1}-G_{n} & =(q-1) G_{n}+(1-q) \alpha_{0} q^{n+1}\left\|Q_{\alpha_{0} q^{n+1}}^{-1} f_{\delta}\right\| \\
& =(1-q)\left(\alpha_{0} q^{n+1}\left\|Q_{\alpha_{0} q^{n+1}}^{-1} f_{\delta}\right\|-G_{n}\right) .
\end{aligned}
$$

Using assumption (44) and applying Lemma 2.6, one gets

$$
G_{n+1}-G_{n}<0, \quad \forall n \geq n_{c} .
$$

Let us prove that the integer $n_{c}$ is unique. Suppose there exists another integer $n_{d}$ such that $G_{n_{d}-1}<G_{n_{d}}$ and $G_{n_{d}}>G_{n_{d}+1}$. One may assume without loss of generality that $n_{c}<n_{d}$. Since $G_{n}>G_{n+1}, \forall n \geq n_{c}$, and $n_{c}<n_{d}$, it follows that $G_{n_{d}-1}>G_{n_{d}}$. This contradicts the assumption $G_{n_{d}-1}<G_{n_{d}}$. Thus, the integer $n_{c}$ is unique. Lemma 2.5 is proved.

Lemma 2.7. Let $G_{n}$ be defined in (34). If $\alpha_{0}$ is chosen such that relations $G_{1}>C \delta^{\varepsilon}, C>1, \varepsilon \in(0,1)$, holds then there exists a unique $n_{\delta}$ satisfying inequality (33).

Proof. Let us show that there exists an integer $n_{\delta}$ so that inequality (33) holds. Applying Lemma 2.4, one gets

$$
\limsup _{n \rightarrow \infty} G_{n} \leq \delta .
$$

Since $G_{1}>C \delta^{\varepsilon}$ and $\limsup _{n \rightarrow \infty} G_{n} \leq \delta<C \delta^{\varepsilon}$, it follows that there exists an index $n_{\delta}$ satisfying stopping rule (33). The uniqueness of the index $n_{\delta}$ follows from the monotonicity of $G_{n}$, see Lemma 2.5. Thus, Lemma 2.7 is proved.

Lemma 2.8. Let $A y=f, y \perp \mathcal{N}(A)$, and $n_{\delta}$ be chosen by rule (33). Then

$$
\lim _{\delta \rightarrow 0} q^{n_{\delta}}=0, \quad q \in(0,1)
$$

so

$$
\lim _{\delta \rightarrow 0} n_{\delta}=\infty
$$


Proof. From rule (33) and relation (34) we have

$$
\begin{aligned}
q C \delta^{\varepsilon}+(1-q) \alpha_{0} q^{n_{\delta}}\left\|Q_{\alpha_{0} q^{n_{\delta}}}^{-1} f_{\delta}\right\| & <q G_{n_{\delta}-1}+(1-q) \alpha_{0} q^{n_{\delta}}\left\|Q_{\alpha_{0} q^{n_{\delta}}}^{-1} f_{\delta}\right\| \\
& =G_{n_{\delta}} \leq C \delta^{\varepsilon}
\end{aligned}
$$

so

$$
(1-q) \alpha_{0} q^{n_{\delta}}\left\|Q_{\alpha_{0} q^{n_{\delta}}}^{-1} f_{\delta}\right\| \leq(1-q) C \delta^{\varepsilon} .
$$

Thus,

$$
\alpha_{0} q^{n_{\delta}}\left\|Q_{\alpha_{0} q^{n_{\delta}}}^{-1} f_{\delta}\right\|<C \delta^{\varepsilon} .
$$

Note that if $f \neq 0$ then there exists a $\lambda_{0}>0$ such that

$$
F_{\lambda_{0}} f \neq 0, \quad\left\langle F_{\lambda_{0}} f, f\right\rangle:=\xi>0,
$$

where $\xi$ is a constant which does not depend on $\delta$, and $F_{s}$ is the resolution of the identity corresponding to the operator $Q:=A A^{*}$. Let

$$
h(\delta, \alpha):=\alpha^{2}\left\|Q_{\alpha}^{-1} f_{\delta}\right\|^{2} .
$$

For a fixed number $c_{1}>0$ we obtain

$$
\begin{aligned}
h\left(\delta, c_{1}\right) & =c_{1}^{2}\left\|Q_{c_{1}} f_{\delta}\right\|^{2}=\int_{0}^{\infty} \frac{c_{1}^{2}}{\left(c_{1}+s\right)^{2}} d\left\langle F_{s} f_{\delta}, f_{\delta}\right\rangle \geq \int_{0}^{\lambda_{0}} \frac{c_{1}^{2}}{\left(c_{1}+s\right)^{2}} d\left\langle F_{s} f_{\delta}, f_{\delta}\right\rangle \\
& \geq \frac{c_{1}^{2}}{\left(c_{1}+\lambda_{0}\right)^{2}} \int_{0}^{\lambda_{0}} d\left\langle F_{s} f_{\delta}, f_{\delta}\right\rangle=\frac{c_{1}^{2}\left\|F_{\lambda_{0}} f_{\delta}\right\|^{2}}{\left(c_{1}+\lambda_{0}\right)^{2}}, \quad \delta>0 .
\end{aligned}
$$

Since $F_{\lambda_{0}}$ is a continuous operator, and $\left\|f-f_{\delta}\right\|<\delta$, it follows from (55) that

$$
\lim _{\delta \rightarrow 0}\left\langle F_{\lambda_{0}} f_{\delta}, f_{\delta}\right\rangle=\left\langle F_{\lambda_{0}} f, f\right\rangle>0 .
$$

Therefore, for the fixed number $c_{1}>0$ we get

$$
h\left(\delta, c_{1}\right) \geq c_{2}>0
$$

for all sufficiently small $\delta>0$, where $c_{2}$ is a constant which does not depend on $\delta$. For example one may take $c_{2}=\frac{\xi}{2}$ provided that (55) holds. Let us derive from estimate (54) and the relation (58) that $q^{n_{\delta}} \rightarrow 0$ as $\delta \rightarrow 0$. From (54) we have

$$
0 \leq h\left(\delta, \alpha_{0} q^{n_{\delta}}\right) \leq\left(C \delta^{\varepsilon}\right)^{2} .
$$

Therefore,

$$
\lim _{\delta \rightarrow 0} h\left(\delta, \alpha_{0} q^{n_{\delta}}\right)=0 .
$$

Suppose $\lim _{\delta \rightarrow 0} q^{n_{\delta}} \neq 0$. Then there exists a subsequence $\delta_{j} \rightarrow 0$ such that

$$
\alpha_{0} q^{n_{\delta_{j}}} \geq c_{1}>0,
$$

where $c_{1}$ is a constant. By (58) we get

$$
h\left(\delta_{j}, \alpha_{0} q^{n_{\delta_{j}}}\right)>c_{2}>0, \quad \delta_{j} \rightarrow 0 \text { as } j \rightarrow \infty .
$$

This contradicts relation (59). Thus, $\lim _{\delta \rightarrow 0} q^{n_{\delta}}=0$. Lemma 2.8 is proved. 
Lemma 2.9. Let $n_{\delta}$ be chosen by rule (33). Then

$$
\frac{\delta}{\sqrt{\alpha_{0} q^{n \delta}}} \rightarrow 0 \text { as } \delta \rightarrow 0 .
$$

Proof. Relation (35), together with stopping rule (33), implies

$$
C \delta^{\varepsilon}<G_{n_{\delta}-1} \leq \frac{1}{1-\sqrt{q}} \frac{\sqrt{\alpha_{0} q^{n_{\delta}-1}}}{2}\|y\|+\delta .
$$

Then

$$
\frac{1}{\sqrt{\alpha_{0} q^{n_{\delta}-1}}} \leq \frac{\|y\|}{2(1-\sqrt{q}) \delta^{\varepsilon}(C-1)}, \quad \varepsilon \in(0,1) .
$$

This yields

$$
\lim _{\delta \rightarrow 0} \frac{\delta}{\sqrt{\alpha_{0} q^{n_{\delta}}}}=\lim _{\delta \rightarrow 0} \frac{\delta}{\sqrt{\alpha_{0} q q^{n_{\delta}-1}}} \leq \lim _{\delta \rightarrow 0} \frac{\delta^{1-\varepsilon}}{2 \sqrt{q}(1-\sqrt{q})(C-1)}\|y\|=0 .
$$

Lemma 2.9 is proved.

Theorem 2.10. Let $y \perp \mathcal{N}(A),\left\|f_{\delta}-f\right\| \leq \delta,\left\|f_{\delta}\right\|>C \delta^{\varepsilon}, C>1, \varepsilon \in(0,1)$. Suppose $n_{\delta}$ is chosen by rule (33). Then

$$
\lim _{\delta \rightarrow 0}\left\|u_{n_{\delta}}^{\delta}-y\right\|=0
$$

where $u_{n}^{\delta}$ is given in (13).

Proof. Using Lemma 2.2 and Lemma 2.3, we get the estimate

$$
\begin{aligned}
\left\|u_{n_{\delta}}^{\delta}-y\right\| & \leq\left\|u_{n_{\delta}}^{\delta}-u_{n_{\delta}}\right\|+\left\|u_{n_{\delta}}-y\right\| \leq \frac{\sqrt{q}}{1-q^{3 / 2}}(1-q) \frac{\delta}{2 q \sqrt{\alpha_{0} q^{n_{\delta}}}}+\left\|u_{n_{\delta}}-y\right\| \\
& :=I_{1}+I_{2},
\end{aligned}
$$

where $I_{1}:=\frac{\sqrt{q}}{1-q^{3 / 2}}(1-q) \frac{\delta}{2 q \sqrt{\alpha_{0} q^{n} \delta}}$ and $I_{2}:=\left\|u_{n_{\delta}}-y\right\|$. Applying Lemma 2.9, one gets $\lim _{\delta \rightarrow 0} I_{1}=0$. Since $n_{\delta} \rightarrow \infty$ as $\delta \rightarrow 0$, it follows from Lemma 2.2 that $\lim _{\delta \rightarrow 0} I_{2}=0$. Thus, $\lim _{\delta \rightarrow 0}\left\|u_{n_{\delta}}^{\delta}-y\right\|=0$. Theorem 2.10 is proved.

The algorithm based on the proposed method can be stated as follows:

Step 1. Assume that (14) holds. Choose $C \in(1,2)$ and $\varepsilon \in(0.9,1)$. Fix $q \in(0,1)$, and choose $\alpha_{0}>0$ so that (17) holds. Set $n=1$, and $u_{0}=0$.

Step 2. Use iterative scheme (13) to calculate $u_{n}$.

Step 3. Calculate $G_{n}$, where $G_{n}$ is defined in (34).

Step 4. If $G_{n} \leq C \delta^{\varepsilon}$ then stop the iteration, set $n_{\delta}=n$, and take $u_{n_{\delta}}^{\delta}$ as the approximate solution. Otherwise set $n=n+1$, and go to Step 1 . 


\section{Iterative scheme 2}

In [8] the following iterative scheme for the exact data $f$ is given:

$$
u_{n+1}=a T_{a}^{-1} u_{n}+T_{a}^{-1} A^{*} f, \quad u_{1}=u_{1} \perp \mathcal{N}(A),
$$

where $a$ is a fixed positive constant. It is proved in [8] that iterative scheme (68) gives the relation

$$
\lim _{n \rightarrow \infty}\left\|u_{n}-y\right\|=0, \quad y \perp \mathcal{N}(A) .
$$

In the case of noisy data the exact data $f$ in $(68)$ is replaced with the noisy data $f_{\delta}$, i.e.

$$
u_{n+1}^{\delta}=a T_{a}^{-1} u_{n}^{\delta}+T_{a}^{-1} A^{*} f_{\delta}, \quad u_{1}=u_{1} \perp \mathcal{N}(A),
$$

where $\left\|f_{\delta}-f\right\| \leq \delta$ for sufficiently small $\delta>0$. It is proved in [8] that there exist an integer $n_{\delta}$ such that

$$
\lim _{\delta \rightarrow 0}\left\|u_{n_{\delta}}^{\delta}-y\right\|=0
$$

where $u_{n}^{\delta}$ is the approximate solution corresponds to the noisy data. But a method of choosing the integer $n_{\delta}$ has not been discussed. In this section we modify iterative scheme (68) by replacing the constant parameter $a$ in (68) with a geometric sequence $\left\{q^{n-1}\right\}_{n=1}^{\infty}$, i.e.

$$
u_{n+1}=q^{n} T_{q^{n}}^{-1} u_{n}+T_{q^{n}}^{-1} A^{*} f, \quad u_{1}=0,
$$

where $q \in(0,1)$. The initial approximation $u_{1}$ is chosen to be 0 . In general one may choose an arbitrary initial approximation $u_{1}$ in the set $\mathcal{N}(A)^{\perp}$. If the data are noisy then the exact data $f$ in $(71)$ is replaced with the noisy data $f_{\delta}$, and iterative scheme (69) is replaced with:

$$
u_{n+1}^{\delta}=q^{n} T_{q^{n}}^{-1} u_{n}^{\delta}+T_{q^{n}}^{-1} A^{*} f_{\delta}, \quad u_{1}^{\delta}=0 .
$$

We prove convergence of the solution obtained by iterative scheme (71) in Theorem 3.1 for arbitrary $q \in(0,1)$, i.e.

$$
\lim _{n \rightarrow \infty}\left\|u_{n}-y\right\|=0, \quad \forall q \in(0,1) .
$$

In the case of noisy data we use discrepancy-type principle (85) to obtain the integer $n_{\delta}$ such that

$$
\lim _{\delta \rightarrow 0}\left\|u_{n_{\delta}}^{\delta}-y\right\|=0
$$

We prove relation (73), for arbitrary $q \in(0,1)$, in Theorem 3.6.

Let us prove that the sequence $u_{n}$, defined by iterative scheme (71), converges to the minimal norm solution $y$ of equation (1).

Theorem 3.1. Consider iterative scheme (71). Let $y \perp \mathcal{N}(A)$. Then

$$
\lim _{n \rightarrow \infty}\left\|u_{n}-y\right\|=0
$$


Proof. Consider the identity

$$
y=a T_{a}^{-1} y+T_{a}^{-1} A^{*} f, \quad A y=f .
$$

Let $w_{n}:=u_{n}-y$ and $B_{n}:=q^{n} T_{q^{n}}^{-1}$. Then $w_{n+1}=B_{n} w_{n}, \quad w_{1}=y-u_{1}=y$. One uses (75) and gets

$$
\begin{aligned}
\left\|y-u_{n}\right\|^{2} & =\left\|B_{n-1} B_{n-2} \ldots B_{1} w_{1}\right\|^{2}=\left\|B_{n-1} B_{n-2} \ldots B_{1} y\right\|^{2} \\
& =\int_{0}^{\infty}\left(\frac{q^{n-1}}{q^{n-1}+s} \frac{q^{n-2}}{q^{n-2}+s} \ldots \frac{q}{q+s}\right)^{2} d\left\langle E_{s} y, y\right\rangle \\
& =\int_{0}^{\infty}\left(\frac{q^{n-1}}{q^{n-1}+s}\right)^{2}\left(\frac{q^{n-2}}{q^{n-2}+s}\right)^{2} \ldots\left(\frac{q}{q+s}\right)^{2} d\left\langle E_{s} y, y\right\rangle \\
& \leq \int_{0}^{\infty} \frac{q^{2 n}}{(q+s)^{2 n}} d\left\langle E_{s} y, y\right\rangle,
\end{aligned}
$$

where $E_{s}$ is the resolution of the identity corresponding to the operator $T:=$ $A^{*} A$. Here we have used the identity (75) and the monotonicity of the function $\phi(x):=\frac{x^{2}}{(x+s)^{2}}, s \geq 0$. From estimate (76) we derive relation (74). Indeed, write

$$
\int_{0}^{\infty} \frac{q^{2 n}}{(q+s)^{2 n}} d\left\langle E_{s} y, y\right\rangle=\int_{0}^{b} \frac{q^{2 n}}{(q+s)^{2 n}} d\left\langle E_{s} y, y\right\rangle+\int_{b}^{\infty} \frac{q^{2 n}}{(q+s)^{2 n}} d\left\langle E_{s} y, y\right\rangle,
$$

where $b$ is a sufficiently small number which will be chosen later. For any fixed $b>0$ one has $\frac{q}{q+s} \leq \frac{q}{q+b}<1$ if $s \geq b$. Therefore, it follows that

$$
\int_{b}^{\infty} \frac{q^{2 n}}{(q+s)^{2 n}} d\left\langle E_{s} y, y\right\rangle \rightarrow 0 \text { as } n \rightarrow \infty .
$$

On the other hand one has

$$
\int_{0}^{b} \frac{q^{2 n}}{(q+s)^{2 n}} d\left\langle E_{s} y, y\right\rangle \leq \int_{0}^{b} d\left\langle E_{s} y, y\right\rangle .
$$

Since $y \perp \mathcal{N}(A)$, one has $\lim _{b \rightarrow 0} \int_{0}^{b} d\left\langle E_{s} y, y\right\rangle=0$. Therefore, given an arbitrary number $\epsilon>0$ one can choose $b(\epsilon)$ such that

$$
\int_{0}^{b(\epsilon)} \frac{q^{2 n}}{(q+s)^{2 n}} d\left\langle E_{s} y, y\right\rangle<\frac{\epsilon}{2} .
$$

Using this $b(\epsilon)$, one chooses sufficiently large $n(\epsilon)$ such that

$$
\int_{b(\epsilon)}^{\infty} \frac{q^{2 n}}{(q+s)^{2 n}} d\left\langle E_{s} y, y\right\rangle<\frac{\epsilon}{2}, \quad \forall n>n(\epsilon) .
$$

Since $\epsilon>0$ is arbitrary, Theorem 3.1 is proved. 
As we mentioned before if the exact data $f$ are contaminated by some noise then iterative scheme (72) is used, where $\left\|f_{\delta}-f\right\| \leq \delta$. Note that

$$
\left\|u_{n+1}^{\delta}-u_{n+1}\right\| \leq q^{n}\left\|T_{q^{n}}^{-1}\left(u_{n}^{\delta}-u_{n}\right)\right\|+\frac{\delta}{2 \sqrt{q^{n}}} \leq\left\|u_{n}^{\delta}-u_{n}\right\|+\frac{\delta}{2 \sqrt{q^{n}}} .
$$

To prove the convergence of the solution obtained by iterative scheme (72), we need the following lemmas:

Lemma 3.2. Let $u_{n}$ and $u_{n}^{\delta}$ be defined in (71) and (72), respectively. Then

$$
\left\|u_{n}^{\delta}-u_{n}\right\| \leq \frac{\sqrt{q}}{1-\sqrt{q}} \frac{\delta}{2 \sqrt{q^{n}}}, \quad n \geq 1 .
$$

Proof. Let us prove relation (83) by induction. For $n=1$ one has $u_{1}^{\delta}-u_{1}=0$. Thus, for $n=1$ the relation holds. Suppose

$$
\left\|u_{l}^{\delta}-u_{l}\right\| \leq \frac{\sqrt{q}}{1-\sqrt{q}} \frac{\delta}{2 \sqrt{q^{l}}}, \quad 1 \leq l \leq k .
$$

Then from (82) and (84) we have

$$
\begin{aligned}
\left\|u_{k+1}^{\delta}-u_{k+1}\right\| & \leq\left\|u_{k}^{\delta}-u_{k}\right\|+\frac{\delta}{2 \sqrt{q^{k}}} \leq \frac{\sqrt{q}}{1-\sqrt{q}} \frac{\delta}{2 \sqrt{q^{k}}}+\frac{\delta}{2 \sqrt{q^{k}}} \\
& \leq \sqrt{q} \frac{\delta}{(1-\sqrt{q}) 2 \sqrt{q^{k+1}}} .
\end{aligned}
$$

Thus,

$$
\left\|u_{n}^{\delta}-u_{n}\right\| \leq \sqrt{q} \frac{\delta}{(1-\sqrt{q}) 2 \sqrt{q^{n}}}, \quad n \geq 1 .
$$

Lemma 3.2 is proved.

Let us formulate our stopping rule: the iteration in iterative scheme (72) is stopped at the first integer $n_{\delta}$ satisfying

$\left\|A T_{q^{n_{\delta}}}^{-1} A^{*} f_{\delta}-f_{\delta}\right\| \leq C \delta^{\varepsilon}<\left\|A T_{q^{n}}^{-1} A^{*} f_{\delta}-f_{\delta}\right\|, \quad 1 \leq n<n_{\delta}, C>1, \varepsilon \in(0,1)$,

and it is assumed that $\left\|f_{\delta}\right\|>C \delta^{\varepsilon}$.

Lemma 3.3. Let $u_{n}^{\delta}$ be defined in (72), and $W_{n}:=\left\|A T_{q^{n}}^{-1} A^{*} f_{\delta}-f_{\delta}\right\|$. Then

$$
W_{n+1} \leq W_{n}, \quad n \geq 1
$$

Proof. Note that

$$
W_{n}=\left\|A A^{*} Q_{q^{n}}^{-1} f_{\delta}-f_{\delta}\right\|=\left\|\left(Q_{q^{n}}-q^{n} I_{m}\right) Q_{q^{n}}^{-1} f_{\delta}-f_{\delta}\right\|=\left\|q^{n} Q_{q^{n}}^{-1} f_{\delta}\right\|,
$$

where $Q:=A A^{*}$, and $Q_{a}:=Q+a I_{m}$. Using the spectral theorem, one gets

$$
W_{n+1}^{2}=\int_{0}^{\infty} \frac{q^{2(n+1)}}{\left(q^{n+1}+s\right)^{2}} d\left\langle F_{s} f_{\delta}, f_{\delta}\right\rangle \leq \int_{0}^{\infty} \frac{q^{2 n}}{\left(q^{n}+s\right)^{2}} d\left\langle F_{s} f_{\delta}, f_{\delta}\right\rangle=W_{n}^{2},
$$


where $F_{s}$ is the resolution of the identity corresponding to the operator $Q:=$ $A A^{*}$. Here we have used the monotonicity of the function $g(x)=\frac{x^{2}}{(x+s)^{2}}, \quad s \geq$ 0 . Thus,

$$
W_{n+1} \leq W_{n}, \quad n \geq 1
$$

Lemma 3.3 is proved.

Lemma 3.4. Let $u_{n}^{\delta}$ be defined in (72), and $\left\|f_{\delta}\right\|>C \delta^{\varepsilon}, \varepsilon \in(0,1), C>1$. Then there exists a unique index $n_{\delta}$ such that inequality (85) holds.

Proof. Let $e_{n}:=A T_{q^{n}}^{-1} A^{*} f_{\delta}-f_{\delta}$. Then

$$
e_{n}=q^{n} Q_{q^{n}}^{-1} f_{\delta},
$$

where $Q_{a}:=A A^{*}+a I$. Therefore,

$$
\begin{aligned}
\left\|e_{n}\right\| & \leq\left\|q^{n} Q_{q^{n}}^{-1}\left(f_{\delta}-f\right)\right\|+\left\|q^{n} Q_{q^{n}}^{-1} f\right\| \\
& \leq\left\|f_{\delta}-f\right\|+\left\|q^{n} Q_{q^{n}}^{-1} A y\right\| \leq \delta+\frac{\sqrt{q^{n}}}{2}\|y\|,
\end{aligned}
$$

where the estimate $\left\|Q_{a}^{-1} A\right\|=\left\|A T_{a}^{-1}\right\| \leq \frac{1}{2 \sqrt{a}}$ was used. Thus,

$$
\limsup _{n \rightarrow \infty}\left\|e_{n}\right\| \leq \delta \text {. }
$$

This shows that the integer $n_{\delta}$, satisfying (85), exists. The uniqueness of $n_{\delta}$ follows from its definition.

Lemma 3.5. Let $u_{n}^{\delta}$ be defined in (72). If $n_{\delta}$ is chosen by rule (85) then

$$
\lim _{\delta \rightarrow 0} \frac{\delta}{\sqrt{q^{n_{\delta}}}}=0 .
$$

Proof. From (91) we have

$$
\left\|A T_{q^{n-1}}^{-1} A^{*} f_{\delta}-f_{\delta}\right\| \leq \delta+\frac{\sqrt{q^{n-1}}}{2}\left\|e_{1}\right\|,
$$

where $e_{1}:=u_{1}-y=-y$. It follows from stopping rule (85) and estimate (93) that

$$
C \delta^{\varepsilon} \leq\left\|A T_{q^{n_{\delta}-1}}^{-1} A^{*} f_{\delta}-f_{\delta}\right\| \leq \frac{\sqrt{q^{n_{\delta}-1}}}{2}\left\|e_{1}\right\|+\delta .
$$

Therefore,

$$
(C-1) \delta^{\varepsilon} \leq \frac{\sqrt{q^{n_{\delta}-1}}}{2}\left\|e_{1}\right\|
$$

and so

$$
\frac{1}{\sqrt{q^{n_{\delta}-1}}} \leq \frac{\left\|e_{1}\right\|}{2(C-1) \delta^{\varepsilon}}, \quad \varepsilon \in(0,1) .
$$


This implies

$$
\frac{\delta}{\sqrt{q^{n_{\delta}}}}=\frac{\delta}{\sqrt{q q^{n_{\delta}-1}}} \leq \frac{\left\|e_{1}\right\| \delta}{2 q^{1 / 2}(C-1) \delta^{\varepsilon}}=\frac{\left\|e_{1}\right\|}{2 q^{1 / 2}(C-1)} \delta^{1-\varepsilon} .
$$

Thus, $\frac{\delta}{\sqrt{q^{n} \delta}} \rightarrow 0$ as $\delta \rightarrow 0$. Lemma 3.5 is proved.

The proof of convergence of the solution obtained by iterative scheme (72) is given in the following theorem:

Theorem 3.6. Let $u_{n}^{\delta}$ be defined in (72), $y \perp \mathcal{N}(A),\left\|f_{\delta}\right\|>C \delta^{\varepsilon}, \varepsilon \in(0,1)$, $C>1, \quad q \in(0,1)$. If $n_{\delta}$ is chosen by rule (85), then

$$
\left\|u_{n}^{\delta}-y\right\| \rightarrow 0 \text { as } \delta \rightarrow 0 .
$$

Proof. From Lemma 3.2 we get the following estimate:

$$
\left\|u_{n_{\delta}}^{\delta}-y\right\| \leq\left\|u_{n_{\delta}}^{\delta}-u_{n_{\delta}}\right\|+\left\|u_{n_{\delta}}-y\right\| \leq \frac{\sqrt{q}}{1-\sqrt{q}} \frac{\delta}{2 \sqrt{q^{n_{\delta}}}}+\left\|u_{n_{\delta}}-y\right\|:=I_{1}+I_{2},
$$

where $I_{1}:=\frac{\sqrt{q}}{1-\sqrt{q}} \frac{\delta}{2 \sqrt{q^{n} \delta}}$ and $I_{2}:=\left\|u_{n_{\delta}}-y\right\|$. By Lemma 3.5 one gets $I_{1} \rightarrow 0$ as $\delta \rightarrow 0$. To prove $\lim _{\delta \rightarrow 0} I_{2}=0$ one needs the relation $\lim _{\delta \rightarrow 0} n_{\delta}=\infty$. This relation is a consequence of the following lemma:

Lemma 3.7. If $n_{\delta}$ is chosen by rule (85), then

$$
q^{n_{\delta}} \rightarrow 0 \text { as } \delta \rightarrow 0
$$

so

$$
\lim _{\delta \rightarrow 0} n_{\delta}=\infty
$$

Proof. Note that

$$
\begin{aligned}
A T_{a}^{-1} A^{*} f_{\delta}-f_{\delta} & =A A^{*} Q_{a}^{-1} f_{\delta}-f_{\delta}=\left(A A^{*}+a I_{m}-a I_{m}\right) Q_{a}^{-1} f_{\delta}-f_{\delta} \\
& =f_{\delta}-a Q_{a}^{-1} f_{\delta}-f_{\delta}=-a Q_{a}^{-1} f_{\delta},
\end{aligned}
$$

where $a>0, Q:=A A^{*}$ and $Q_{a}:=Q+a I$. From stopping rule (85) we have $0 \leq\left\|A T_{q^{n} \delta}^{-1} A^{*} f_{\delta}-f_{\delta}\right\| \leq C \delta^{\varepsilon}$. Thus,

$$
\lim _{\delta \rightarrow 0}\left\|A T_{q^{n_{\delta}}}^{-1} A^{*} f_{\delta}-f_{\delta}\right\|=\lim _{\delta \rightarrow 0}\left\|q^{n_{\delta}} Q_{q^{n_{\delta}}}^{-1} f_{\delta}\right\|=0 .
$$

Using an argument given in the proof of Lemma 2.8, (see formulas (54)-(61) in which $\alpha_{0}=1$ ), one gets $\lim _{\delta \rightarrow 0} q^{n_{\delta}}=0$, so $\lim _{\delta \rightarrow 0} n_{\delta}=\infty$. Lemma 3.7 is proved.

Lemma 3.7 and Theorem 3.1 imply $I_{2} \rightarrow 0$ as $\delta \rightarrow 0$. Thus, Theorem 3.6 is proved. 


\section{Numerical experiments}

In all the experiments we measure the accuracy of the approximate solutions using the relative error:

$$
\text { Rel.Err }=\frac{\left\|u_{n_{\delta}}^{\delta}-y\right\|}{\|y\|}
$$

where $\|$.$\| is the Euclidean norm in \mathbb{R}^{n}$. The exact data are perturbed by some noises so that

$$
\left\|f_{\delta}-f\right\| \leq \delta
$$

where

$$
f_{\delta}=f+\delta \frac{e}{\|e\|},
$$

$\delta$ is the noise level, and $e \in \mathbb{R}^{n}$ is the noise taken from the Gaussian distribution with mean 0 and standard deviation 1 . The MATLAB routine called "randn" with seed 15 is used to generate the vector $e$. The iterative schemes (13) and (72) will be denoted by $I S_{1}$ and $I S_{2}$, respectively. In the iterative scheme $I S_{1}$, for fixed $q \in(0,1)$, one needs to choose a sufficiently large $\alpha_{0}>0$ so that inequality (17) hold, for example one may choose $\alpha_{0} \geq 1$. The number of iterations of $I S_{1}$ and $I S_{2}$ are denoted by Iter 1 and Iter 2 , respectively. We compare the results obtained by the proposed methods with the results obtained by using the variational regularization method (VR). In VR we use the Newton method for solving the equation for regularization parameter. In [4] the nonlinear equation

$$
\left\|A u_{V R}(a)-f_{\delta}\right\|^{2}=(C \delta)^{2}, C=1.01,
$$

where $u_{V R}(a):=T_{a}^{-1} A^{*} f_{\delta}$, is solved by the Newton's method. In this paper the initial value of the regularization parameter is taken to be $\alpha_{0}=\frac{\alpha_{0}}{2^{k} \delta}$, where $k_{\delta}$ is the first integer such that the Newton's method for solving (103) converges. We stop the iteration of the Newton's method at the first integer $n_{\delta}$ satisfying the inequality $\left|\left\|A T_{a_{n}}^{-1} A^{*} f_{\delta}-f_{\delta}\right\|^{2}-(C \delta)^{2}\right| \leq 10^{-3}(C \delta)^{2}, \quad a_{0}:=\alpha_{0}$. The number of iterations needed to complete a convergent Newton's method is denoted by Iter $_{V R}$.

\subsection{Ill-conditioned linear algebraic systems}

Table 1: Condition number of some Hilbert matrices

\begin{tabular}{ll}
\hline$n$ & $\kappa(A)=\|A\|\left\|A^{-1}\right\|$ \\
\hline 10 & $1.915 \times 10^{13}$ \\
20 & $1.483 \times 10^{28}$ \\
70 & $8.808 \times 10^{105}$ \\
100 & $1.262 \times 10^{150}$ \\
200 & $1.446 \times 10^{303}$ \\
\hline
\end{tabular}


Consider the following system:

$$
H^{(m)} u=f,
$$

where

$$
H_{i j}^{(m)}=\frac{1}{i+j+1}, \quad i, j=1,2, \ldots, m,
$$

is a Hilbert matrix of dimension $m$. The system (104) is an example of a severely ill-posed problem if $m>10$, because the condition number of the Hilbert matrix is increasing exponentially as $m$ grows, see Table (1). The minimal eigenvalues of Hilbert matrix of dimension $m$ can be obtained using the following formula

$$
\lambda_{\min }\left(H^{(m)}\right)=2^{15 / 4} \pi^{3 / 2} \sqrt{m}(\sqrt{2}+1)^{-(4 m+4)}(1+o(1)) .
$$

This formula is proved in [3]. Since $\kappa\left(H^{(m)}\right)=\frac{\lambda_{\max }\left(H^{(m)}\right)}{\lambda_{\min }\left(H^{(m)}\right)}$, it follows from (105) that the condition number grows as $O\left(\frac{e^{3.5255 m}}{\sqrt{m}}\right)$. The following exact solution is used to test the proposed methods:

$$
y \in \mathbb{R}^{m} \text {, where } y_{k}=\sqrt{.5 k}, k=1,2, \ldots, m .
$$

The Hilbert matrix of dimension $m=200$ is used in the experiments. This matrix has condition number of order $10^{303}$, so it is a severely ill-conditioned matrix. In Table 2 one can see that the number of iterations of the iterative

Table 2: Hilbert matrix problem: the number of iterations and the relative errors with respect to the parameter $q\left(\alpha_{0}=1, \quad \delta=10^{-2}\right)$.

\begin{tabular}{lllll}
\hline$q$ & \multicolumn{2}{c}{ IS $_{1}$} & \multicolumn{2}{c}{ IS $_{2}$} \\
\hline & REl.Err & Iter $_{1}$ & REl.Err & Iter $_{2}$. \\
\hline .5 & 0.031 & 24 & 0.032 & 23 \\
.25 & 0.031 & 13 & 0.032 & 13 \\
.125 & 0.032 & 9 & 0.032 & 9 \\
\hline
\end{tabular}

scheme $I S_{1}$ and $I S_{2}$ increases as the value of $q$ increases. The relative errors start to increase at $q=.125$. By these observations, we suggest to choose the parameter $q$ in the interval $(.125, .5)$. In Table 3 the results of the experiments with various values of $\delta$ are presented. Here the parameter $\varepsilon$ was .99. The geometric sequence $\left\{.25^{n-1}\right\}_{n=1}^{\infty}$ was used in the iterative schemes $I S_{1}$ and $I S_{2}$. The parameter $C$ in (16) and (85) were 1.01. The parameter $k_{\delta}$ in the variational regularization method was 1 . One can see that the relative errors of $I S_{1}$ and $I S_{2}$ are smaller than these for the VR. The relative error decreases as the noise level decreases which can be seen on the same table. This shows that the proposed method produces stable solutions. 
Table 3: ICLAS with Hilbert matrix: the relative errors and the number of iterations

\begin{tabular}{lllllll}
\hline$\delta$ & \multicolumn{2}{c}{ IS $_{1}$} & \multicolumn{2}{c}{ IS $_{2}$} & \multicolumn{2}{c}{ VR } \\
\hline & REl.Err & Iter $_{1}$ & REl.Err & Iter $_{2}$ & REl.Err & Iter $_{V R}$. \\
\hline $5 \%$ & 0.038 & 11 & 0.043 & 11 & 0.055 & 13 \\
$3 \%$ & 0.037 & 12 & 0.034 & 12 & 0.045 & 14 \\
$1 \%$ & 0.031 & 13 & 0.032 & 13 & 0.034 & 15 \\
\hline
\end{tabular}

\subsection{Fredholm integral equations of the first kind (FIEFK)}

Here we consider two Fredholm integral equations :

a)

$$
f(s)=\int_{-3}^{3} k(t-s) u(t) d t
$$

where

$$
k(z)= \begin{cases}1+\cos \left(\frac{\pi}{3} z\right), & |z|<3 \\ 0, & |z| \geq 3\end{cases}
$$

and

$$
f(z)= \begin{cases}(6+z)\left[1-\frac{1}{2} \cos \left(\frac{\pi}{3} z\right)\right]-\frac{9}{2 \pi} \sin \left(\frac{\pi z}{3}\right), & |z| \leq 6 \\ 0, & |z|>6 .\end{cases}
$$

b)

$$
f(s)=\int_{0}^{1} k(s, t) u(t) d t, s \in(0,1)
$$

where

$$
k(s, t)= \begin{cases}s(t-1), & s<t \\ t(s-1), & s \geq t\end{cases}
$$

and

$$
f(s)=\left(s^{3}-s\right) / 6 .
$$

The problem $a$ ) is discussed in [5] where the solution to this problem is $u(x)=$ $k(x)$. The second problem is taken from [1] where the solution is $u(x)=x$. The Galerkin's method is used to discretized the integrals (106) and (109). For the basis functions we use the following orthonormal box functions

$$
\phi_{i}(s):= \begin{cases}\sqrt{\frac{m}{c_{1}}}, & {\left[s_{i-1}, s_{i}\right]} \\ 0, & \text { otherwise }\end{cases}
$$

and

$$
\psi_{i}(t):= \begin{cases}\sqrt{\frac{m}{c_{2}}}, & {\left[t_{i-1}, t_{i}\right]} \\ 0, & \text { otherwise }\end{cases}
$$


where $s_{i}=d_{1}+i \frac{d_{2}}{m}, t_{i}=d_{3}+i \frac{d_{4}}{m}, i=0,1,2, \ldots, m$. In the problem $\left.a\right)$ the parameters $c_{1}, c_{2}, d_{1}, d_{2}, d_{3}$ and $d_{4}$ are set to $12,6,-6,12,-3$ and 6 , respectively. In the second problem we use $d_{1}=d_{3}=0$ and $c_{1}=c_{2}=d_{2}=$ $d_{4}=1$. Here we approximate the solution $u(t)$ by $\tilde{u}=\sum_{j=1}^{m} c_{j} \psi_{j}(t)$. Therefore solving problem (106) is reduced to solving the linear algebraic system

$$
A \tilde{c}=f, \tilde{c}, f \in \mathbb{R}^{m},
$$

where in problem $a$ )

$$
A_{i j}=\int_{-3}^{3} \int_{-6}^{6} k(t-s) \phi_{i}(s) \psi_{j}(t) d s d t
$$

and $f_{i}=\int_{-6}^{6} f(s) \phi_{i}(s) d s, i, j=1,2, \ldots, m$, and in problem $\left.b\right)$

$$
A_{i j}=\int_{0}^{1} \int_{0}^{1} k(s, t) \phi_{i}(s) \psi_{j}(t) d s d t
$$

and $f_{i}=\int_{0}^{1} f(s) \phi_{i}(s) d s$, and $\tilde{c}_{j}=c_{j} i, j=1,2, \ldots, m$.

Table 4: Problem $a)$ : the number of iterations and the relative errors with respect to the parameter $q\left(\alpha_{0}=2, \quad \delta=10^{-2}\right)$.

\begin{tabular}{lllll}
\hline$q$ & \multicolumn{2}{c}{ IS $_{1}$} & \multicolumn{2}{c}{ IS $_{2}$} \\
\hline & REl.Err & Iter $_{1}$ & REl.Err & Iter $_{2}$. \\
\hline .5 & 0.008 & 12 & 0.007 & 11 \\
.25 & 0.009 & 7 & 0.007 & 7 \\
.125 & 0.009 & 5 & 0.008 & 5 \\
\hline
\end{tabular}

Table 5: Problem a): the relative errors and the number of iterations

\begin{tabular}{cllllll}
\hline$\delta$ & \multicolumn{2}{c}{$I S_{1}$} & \multicolumn{2}{c}{$I S_{2}$} & \multicolumn{2}{c}{$\mathrm{VR}$} \\
\hline & REl.Err & Iter $_{1}$ & REl.Err & Iter $_{2}$ & REl.Err & Iter $_{V R}$. \\
\hline $5 \%$ & 0.018 & 6 & 0.014 & 6 & 0.016 & 11 \\
$3 \%$ & 0.013 & 6 & 0.011 & 6 & 0.013 & 12 \\
$1 \%$ & 0.009 & 7 & 0.007 & 7 & 0.008 & 15 \\
\hline
\end{tabular}

The parameter $m=600$ is used in problem $a$ ). In this case the condition number of the matrix $A$ with $m=600$ is $3.427 \times 10^{9}$, so it is an ill-conditioned matrix. Here the parameter $C$ in $I S_{1}$ and $I S_{2}$ are 2 and 1.01, respectively. For problem $b$ ) the parameter $m$ is 200 . In this case the condition number of the 
Table 6: Problem $b$ ): the number of iterations and the relative errors with respect to the parameter $q\left(\alpha_{0}=4, \quad \delta=10^{-2}\right)$.

\begin{tabular}{lllll}
\hline$q$ & \multicolumn{2}{c}{ IS $_{1}$} & \multicolumn{2}{c}{ IS $_{2}$} \\
\hline & REl.Err & Iter $_{1}$ & REl.Err & Iter $_{2}$. \\
\hline .5 & 0.428 & 17 & 0.446 & 15 \\
.25 & 0.421 & 9 & 0.436 & 9 \\
.125 & 0.439 & 6 & 0.416 & 7 \\
\hline
\end{tabular}

Table 7: Problem $b$ ): the relative errors and the number of iterations

\begin{tabular}{lllllll}
\hline$\delta$ & \multicolumn{2}{c}{$I_{1}$} & \multicolumn{2}{c}{ IS $_{2}$} & \multicolumn{2}{c}{ VR } \\
\hline & REl.Err & Iter $_{1}$ & REl.Err & Iter $_{2}$ & REl.Err & Iter $_{V}$. \\
\hline $5 \%$ & 0.618 & 7 & 0.621 & 7 & 0.627 & 12 \\
$3 \%$ & 0.541 & 8 & 0.559 & 8 & 0.584 & 13 \\
$1 \%$ & 0.421 & 9 & 0.436 & 9 & 0.457 & 13 \\
\hline
\end{tabular}

matrix $A$ is $4.863 \times 10^{4}$. The parameter $C$ is 1.01 in the both iterative schemes $I S_{1}$ and $I S_{2}$. In Tables 4 and 6 we give the relation between the parameter $q$ and the number of iterations and the relative errors of the iterative schemes $I S_{1}$ and $I S_{2}$. The closer the parameter $q$ to 1 , the larger number of iterations we get, and the closer the parameter $q$ to 0 , the smaller the number of iterations we get. But the relative error starts to increase if the parameter $q$ is chosen too small. Based on the numerical results given in Tables 4 and 5 , we suggest to choose the parameter $q$ in the interval $(0.125,0.5)$. In the iterative schemes $I S_{1}$ and $I S_{2}$ we use the geometric sequence $\left\{2 \times .25^{n-1}\right\}_{n=1}^{\infty}$ for problem a). The geometric series $\left\{4 \times .25^{n-1}\right\}_{n=1}^{\infty}$ is used in problem b). In the variational regularization method we use $\alpha_{0}=2$ and $\alpha_{0}=4$ as the initial regularization parameter of the Newton's method in problem $a$ ) and $b$ ), respectively. Since the Newton's method for solving (103) is locally convergent, in problem $b$ ) we need to choose a smaller regularization parameter $\alpha_{0}$ than for $I S_{1}$ and $I S_{2}$ methods. Here $k_{\delta}=8$ was used. The numerical results on Table 5 show that the solutions produced by the proposed iterative schemes are stable. In problem a) the relative errors of the iterative scheme $I S_{2}$, are smaller than these for the iterative scheme $I S_{1}$ and than these for the variational regularization, VR. In Table 7 the relative errors produced by the three methods for solving problem $b)$ are presented. The relative error of $I S_{1}$ is smaller than the one for the other two methods. 


\section{Conclusion}

We have demonstrated that the proposed iterative schemes can be used for solving ill-conditioned linear algebraic systems stably. The advantage of the iterative scheme (13) compared with iterative scheme (72) is the following: one applies the operator $T_{a}^{-1}$ only once at each iteration. Note that the difficulty of using the Newton's method is in choosing the initial value for the regularization parameter, since the Newton's method for solving equation (103) converges only locally. In solving (103) by the Newton's method one often has to choose an initial regularization parameter $a_{0}$ sufficiently close to the root of equation (103) as shown in problem $b$ ) in Section 4.2. In our iterative schemes the initial regularization parameter can be chosen in the interval $[1,4]$ which is larger than the initial regularization parameter used in the variational regularization method. In the iterative scheme $I S_{1}$ we modified the discrepancy-type principle

$$
\int_{0}^{t} e^{-(t-s)} a(s)\left\|Q_{a(s)}^{-1}\right\| d s=C \delta, C \in(1,2),
$$

given in [11], by using (10) to get discrepancy-type principle (33), which can be easily implemented numerically. In Section 3 we used the geometric series $\left\{\alpha_{0} q^{n}\right\}_{n=1}^{\infty}$ in place of the constant regularization parameter $a$ in the iterative scheme

$$
u_{n+1}=a T_{a}^{-1} u_{n}+T_{a}^{-1} A^{*} f_{\delta}
$$

developed in [8]. This geometric series of the regularization parameter allows one to use the a posteriori stopping rule given in (85). We proved that this stopping rule produces stable approximation of the minimal norm solution of equation (1). In all the experiments stopping rules (33) and (85) produce stable approximations to the minimal norm solution of equation (1). It is of interest to develop a method for choosing the parameter $q$ in the proposed methods which gives sufficiently small relative error and small number of iterations.

\section{References}

[1] Delves, L. M. and Mohamed, J. L., Computational Methods for Integral Equations, Cambridge University Press, 1985; p. 310.

[2] Hoang, N.S. and Ramm, A. G. Solving ill-conditioned linear algebraic systems by the dynamical systems method (DSM), Inverse Problems in Sci. and Engineering, 16, N5, (2008), 617-630.

[3] Kalyabin, G. A., An asymptotic formula for the minimal eigenvalues of Hilbert type matrices, Functional analysis and its applications, Vol. 35, No. 1, pp 67-70, 2001.

[4] Morozov, V., Methods of solving incorrectly posed problems, Springer Verlag, New York, 1984. 
[5] Phillips, D. L., A technique for the numerical solution of certain integral equations of the first kind, J Assoc Comput Mach, 9, 84-97, 1962.

[6] Ramm, A. G., Inverse problems, Springer, New York, 2005.

[7] Ramm, A. G., Ill-posed problems with unbounded operators, Journ. Math. Anal. Appl., 325, , 490-495,2007.

[8] Ramm, A. G., Iterative solution of linear equations with unbounded operators, Jour. Math. Anal. Appl., 330, N2, 1338-1346, 2007.

[9] Ramm, A. G., Dynamical systems method for solving operator equations, Elsevier, Amsterdam, 2007.

[10] Ramm, A. G., On unbounded operators and applications, Appl. Math. Lett., 21, 377-382, 2008.

[11] Ramm, A. G., Discrepancy principle for DSM, I, II, Comm. Nonlin. Sci. and Numer. Simulation, 10, N1, (2005), 95-101; 13, (2008), 1256-1263.

[12] Ramm, A. G., A new discrepancy principle, Journ. Math.Anal. Appl., 310, (2005), 342-345. 